\title{
Die Präzisierung des Inflationsziels - ein Ausweg aus der Niedrigzinspolitik?
}

Nach dem Ausbruch der Finanzkrise haben Zentralbanken weltweit nicht nur ihre Leitzinsen drastisch gesenkt, sie probierten auch mit ungeahnter Kreativität den Einsatz einer Vielzahl ganz unkonventioneller Maßnahmen aus - angefangen von der massiven Ausweitung der Zentralbankbilanzen (durch den Aufkauf meist von Staatsanleihen, teilweise aber auch von Unternehmensanleihen und Aktienpaketen) über gezielte Kommunikationsstrategien (Forward Guidance) des zukünftig geplanten Politikpfads bis hin zu Experimenten mit negativen Leitzinsen. Trotz der ungewöhnlich lang anhaltenden Periode lockerer Geldpolitik hat sich das Wirtschaftswachstum bislang jedoch kaum erholt; auch die Inflationsrate verharrt in den meisten Industriestaaten auf historisch niedrigen Werten.

\section{Kritik an der EZB-Politik}

Gerade die Politik der Europäischen Zentralbank (EZB) sieht sich mittlerweile vor allem in der deutschen Öffentlichkeit immer stärkerer Kritik ausgesetzt. Viele werfen ihr vor, die Niedrigzinspolitik „enteigne“ den deutschen Sparer. Sie fordern vehement einen raschen Ausstieg aus der Niedrigzinspolitik und warnen vor negativen Auswirkungen der ultraniedrigen Zinspolitik auf das gesamte Finanzsystem. Manche argumentieren, nur durch eine zügige Anhebung der Zinsen ließe sich der nötige Spielraum schaffen, um zukünftig im Fall eines Abschwungs die Zinsen wieder senken zu können.

Die Kritiker übersehen dabei, dass das Zinsniveau weltweit in ungeahnte Tiefen gefallen ist. Niedrige Zinsen sind keineswegs ein Sonderweg der EZB, sondern die „neue Normalität" in den Industriestaaten. Ende 2015 begann die US-amerikanische Zentralbank Fed mit einer Reihe von kleinen Zinsschritten nach oben mit dem Versuch, eine Normalisierung einzuleiten - sie erhöhte den Leitzins langsam von $0,25 \%$ bis auf $2,5 \%$ im Dezember 2018. Doch schon im August 2019 leitete die Fed wieder eine weitere Zinssenkungsrunde ein. Die Forschungsabteilungen der Fed führen derzeit zudem eine intensive Diskussion zur Überprüfung ihrer geldpolitischen Strategie, Instrumente und Kommuni-

(C) Der/die Autor(en) 2020. Open Access: Dieser Artikel wird unter der Creative Commons Namensnennung 4.0 International Lizenz (https:// creativecommons.org/licenses/by/4.0/deed.de) veröffentlicht.

Open Access wird durch die ZBW - Leibniz-Informationszentrum Wirtschaft gefördert. kationspolitik, um besser gerüstet zu sein, falls die Zinsen wieder an die Untergrenze stoßen.

Während die US-amerikanische Zentralbank ebenso wie auch die Bank of England aus Sorge um die Stabilität des Finanzsektors bewusst darauf verzichtete, den Leitzins negativ werden zu lassen, wagten andere (zunächst Dänemark, ab Anfang 2015 dann die EZB - mit negativem Einlagenzins -, die Schweiz sowie Schweden und schließlich auch Japan) das Experiment, zu testen, wie tief die Zinsuntergrenze unter null liegen könnte. Die Schweizer Nationalbank ging dabei am weitesten. Ihr Leitzins liegt seit Januar 2015 unverändert bei $-0,75 \%$. Sie schließt derzeit nicht aus, dass unter Umständen selbst eine weitere Lockerung notwendig werden könnte. Dagegen hat sich die Schwedische Reichsbank im letzten Monat von der Phase negativer Leitzinsen wieder verabschiedet: Am 19. Dezember 2019 hob sie - trotz einer Abschwächung der schwedischen Wirtschaft - ihren Leitzins wieder auf $0 \%$ an, nachdem sie inn mehrere Jahre lang negativ gesetzt hatte. Sie begründete diesen Schritt mit der Befürchtung, zu lang anhaltende negative Zinsen könnten unerwünschte Verhaltensänderungen mit negativen Folgen auslösen. Die Schwedische Reichsbank betonte aber zugleich, der Leitzins werde wohl auf Jahre hinaus bei null verharren und schloss auch eine künftige Lockerung keineswegs aus.

\section{Grenzen der Niedrigzinspolitik}

Ganz offensichtlich stoßen die Zentralbanken weltweit genau an die Grenzen, die von der makroökonomischen Theorie schon seit John Maynard Keynes eingehend charakterisiert wurden: Durch Anpassung des kurzfristigen Leitzinses im Konjunkturverlauf kann Geldpolitik im Normalfall erfolgreich zu einer Stabilisierung von Inflation und Produktionsschwankungen beitragen. In den vergangenen Jahrzehnten senkten die Zentralbanken im Lauf eines Konjunkturabschwungs ihre Leitzinsen im Durchschnitt in der Regel um etwa 5 Prozentpunkte. Der Spielraum für solche

Prof. Dr. Gerhard Illing leitet das Seminar für Makroökonomie am Institut für Volkswirtschaftslehre der Ludwig-Maximilians-Universität München. 
Zinssenkungen ist jedoch dadurch begrenzt, dass der Nominalzins nicht stark unter null sinken kann, ohne eine Flucht in das Horten von Bargeld auszulösen und damit die Stabilität des Bankensektors zu gefährden. Sobald Geldpolitik diese effektive Zinsuntergrenze erreicht, greifen die traditionellen Instrumente nicht mehr wie gewohnt. Mangelnde gesamtwirtschaftliche Nachfrage schlägt sich dann letztlich in einer Unterauslastung der Ressourcen nieder, sofern der Nachfrageausfall nicht durch stützende aktive Fiskalpolitik kompensiert wird.

Schon Keynes war sich bewusst, dass diese Zinsuntergrenze angesichts von Hortungskosten keineswegs bei null liegt, sondern leicht darunter. Der Anreiz zum Horten lässt sich zudem abmildern, wenn nur zusätzliche Bankeinlagen mit einem marginalen negativen Zinssatz belastet werden, bestehende Einlagen dagegen davon ausgenommen bleiben. So verfährt man in der Schweiz und in Japan schon seit Einführung negativer Zinsen; im Euroraum erst seit September 2019. Geht es zudem - wie im Fall der Schweiz - darum, Anlagen im eigenen Land für internationale Investoren unattraktiv zu machen, mag die Untergrenze noch tiefer liegen. Solange es Bargeld gibt, lässt sie sich aber nicht wegzaubern. Moderne Ansätze ${ }^{1}$ modellieren diesen Sachverhalt eleganter als „Reversal Rate“ unterhalb derer die Kreditvergabe angesichts rückläufiger Profitabilität des Bankensektors zurückgeht und sich so der Nachfrageausfall verstärkt. Der grundlegende Mechanismus bleibt davon aber unberührt.

Die neue Einsicht aus den Erfahrungen der Finanzkrise besteht vielmehr darin, dass das Problem mangelnder Nachfrage sehr lange andauern kann, sofern nicht von Anfang an entschieden gegengesteuert wird. Die Gefahr einer säkularen Stagnation ist zu einem beängstigend realistischen Szenario geworden. Dieser Aspekt dürfte bei der Überprüfung der geldpolitischen Strategie der EZB, die jüngst von der neuen Chefin Christine Lagarde angestoßen wurde, eine wichtige Rolle spielen.

\section{Spiegelbild realer langfristiger Entwicklungen?}

Moderne makroökonomische Theorieansätze liefern eine eindeutige Antwort auf die Forderung nach einem raschen Ausstieg aus der Niedrigzinspolitik: Die derzeit niedrigen Zinsen sind keineswegs das Resultat einer fahrlässigen Politik verantwortungsloser Zentralbanker; sie sind vielmehr Spiegelbild realer langfristiger Entwicklungen - dem stetigen Rückgang realer Wachstumsraten (und damit des „natürlichen“ Realzinses) sowie dem stetigen Rückgang der Inflation (und damit auch der Inflationserwartungen) in den

1 Etwa von M. Brunnermeier, Y. Koby: The Reversal Interest Rate, mimeo, Princeton University, 2019, https://scholar.princeton.edu/sites/ default/files/markus/files/25b_reversalrate.pdf (9.1.2020).
Abbildung 1

Rendite zehnjähriger Staatsanleihen

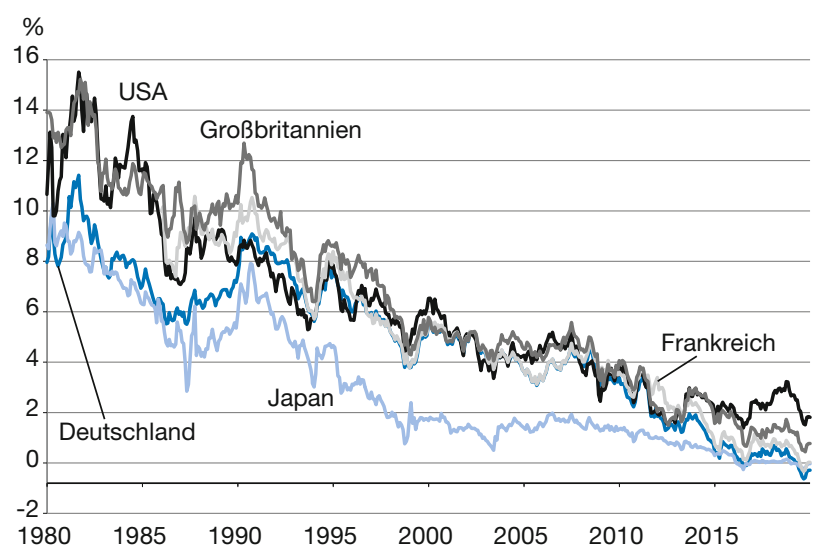

Quelle: Thomson Reuters.

meisten Industriestaaten. Ein zu rascher Ausstieg würde die Gefahr verschärfen, in einem Szenario langfristiger säkularer Stagnation zu verharren. Ein einfacher Blick auf die Entwicklung der nominalen Renditen von Staatsanleihen mit einer Laufzeit von zehn Jahren über die letzten 40 Jahre (vgl. Abbildung 1) verdeutlicht den grundlegenden Mechanismus. Über den gesamten betrachteten Zeitraum sind die Renditen stark gesunken.

Der weltweite Rückgang lässt sich kaum als Resultat einer Manipulation verschwörerischer Zentralbanken interpretieren; er ist ökonomisch vielmehr ganz einfach zu erklären: Auf mittlere bis langfristige Sicht muss für den Nominalzins die bereits 1930 von Irving Fisher formulierte Arbitragegleichung $i=r+\pi^{e}$ erfüllt sein. Langfristig bestimmt sich der gleichgewichtige Nominalzins $i_{n}=r_{n}+\pi^{e}$ dabei als Summe aus der erwarteten Inflationsrate $\pi^{e}$ und dem „natürlichen“ Realzins $r_{n}$ der Wirtschaft.

Der Rückgang der Inflationserwartungen lässt sich unmittelbar auf den Erfolg der Zentralbanken bei der Bekämpfung hoher Inflationsraten seit den 1970er Jahren des letzten Jahrhunderts zurückführen (vgl. Abbildung 2). Er zeigt sich direkt in niedrigeren Aufschlägen auf den geforderten Realzins als Kompensation des Inflationsrisikos. In Deutschland und Frankreich ist die Nominalverzinsung zehnjähriger Staatsanleihen derzeit leicht negativ. Würden die Finanzmärkte im Lauf der kommenden zehn Jahre mit stark steigenden Inflationsraten rechnen, müsste sich dies in steigenden Renditen langfristiger Nominalanleihen widerspiegeln.

\section{Der natürliche Realzins}

Auch der „natürliche“ Realzins ist im Laufe der vergangenen Jahrzehnte gesunken. Zum Rückgang des Realzinses tragen 
Abbildung 2

Rückgang der Inflationsraten seit den 1970er Jahren

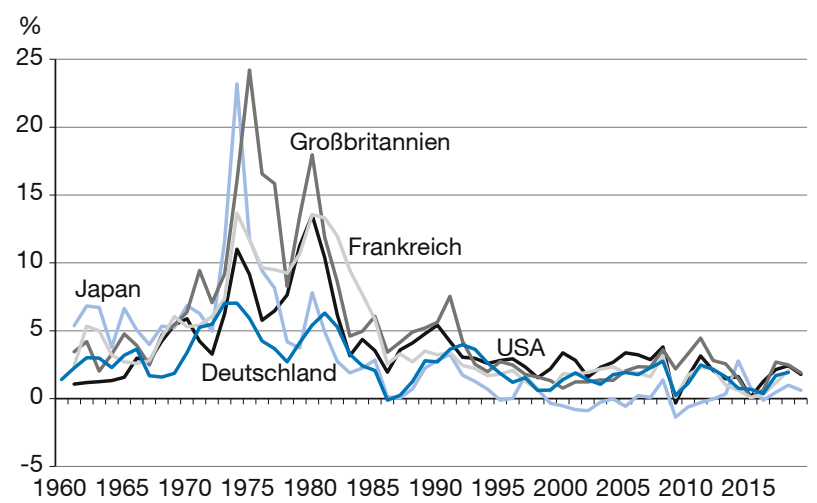

Quelle: OECD Economic Outlook.

ganz unterschiedliche Faktoren bei, wie das abnehmende Trendwachstum der Produktivität, ein Anstieg der Sparneigung aufgrund der demografischen Bevölkerungsstruktur, zunehmende Risikoaversion, die mit steigender Nachfrage nach sicheren Staatsanleihen einhergeht, sowie ein Anstieg von privater Verschuldung und Ungleichheit, wenn ärmere verschuldete Bevölkerungsschichten weniger sparen als Reiche, die aufgrund von Statuspräferenzen oder Vererbungsmotiven Vermögen zu akkumulieren suchen. ${ }^{2}$ Andere Beiträge des aktuellen Zeitgesprächs (insbesondere von Weizsäcker) ${ }^{3}$ analysieren einige dieser Faktoren ausführlicher.

Das Konzept ist (analog zur strukturell bedingten Arbeitslosenquote und der Wachstumsrate des Produktionspotenzials) ein theoretisches Konstrukt und somit empirisch nur schwer messbar. Abbildung 3 zeigt die Bandbreite aktueller Schätzungen von Brand und Mazelis zum Verlauf des natürlichen Realzinses im Euroraum. ${ }^{4}$ Schätzungen der wahren Rate sind immer mit hoher Unsicherheit behaftet. Aber der in Abbildung 3 erkennbare Abwärtstrend erweist sich über ganz unterschiedliche Schätzverfahren hinweg als erstaunlich robust.

Geldpolitik hat auf den natürlichen Realzins $r_{n}$ keinen direkten Einfluss. Ihre Aufgabe besteht vielmehr darin, durch Anpassungen des kurzfristigen Nominalzinses im Konjunkturverlauf dafür zu sorgen, dass der aktuelle Realzins dem natürlichen Zins entspricht und so sicherzustellen, dass die

2 Vgl. dazu G. Illing, Y. Ono, M. Schlegl: Credit Booms, Debt Overhang and Secular Stagnation, in: European Economic Review, 108. Jg. (2018), S. 78-104; A. Mian, L. Straub, A. Sufi: Indebted Demand, Working Paper, 2019, https://scholar.harvard.edu/files/straub/files/ mss_indebteddemand.pdf (9.1.2020).

3 C. von Weizsäcker: Ende der Kapitalknappheit und neuer Protektionismus, Beitrag zum Zeitgespräch im Wirtschaftsdienst, in: Wirtschaftsdienst, 100. Jg. (2020), H. 1, S. 25-28.

4 C. Brand, F. Mazelis: Taylor-rule consistent estimates of the natural rate of interest, ECB, Working Paper Series, Nr. 2257, 2019.
Abbildung 3

Rückgang des natürlichen Realzinses im Euroraum Bandbreite der Schätzungen

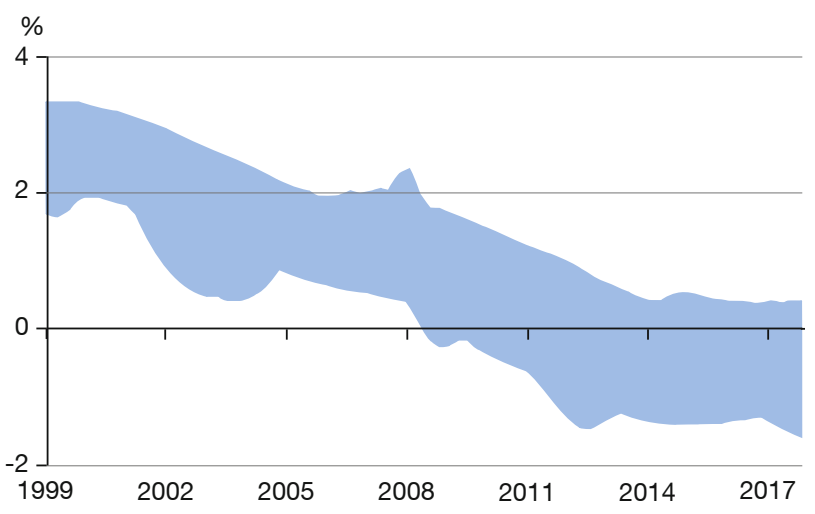

Quelle: C. Brand, F. Mazelis: Taylor-rule consistent estimates of the natural rate of interest, ECB, Working Paper Series, Nr. 2257, 2019.

tatsächliche Inflation in Einklang mit den Inflationserwartungen steht. Ist etwa der Realzins zu hoch $\left(r>r_{n}\right)$, so fällt die gewünschte Ersparnis im Vergleich zu den geplanten Investitionen ebenfalls zu hoch aus. Dann wäre eine Zinssenkung angebracht, um eine zu niedrige gesamtwirtschaftliche Nachfrage wieder in Einklang mit dem Produktionspotenzial zu bringen. Umgekehrt wäre im Fall $r<r_{n}$ ein Anstieg der Zinsen angemessen. Angesichts der Gültigkeit sowohl der Fisher-Gleichung als auch einer effektiven Zinsuntergrenze sieht sich die Zentralbank aber mit folgender Asymmetrie konfrontiert: Wegen $i=r+\pi^{e} \geq 0$ kann der Realzins nicht allzu stark sinken: $r \geq-\pi^{e}$. Je niedriger der natürliche Realzins $r_{n}$ bzw. je niedriger die Inflationserwartungen, desto häufiger wird folglich der Handlungsspielraum konventioneller Geldpolitik durch die effektive Zinsuntergrenze eingeengt. Gerade in Zeiten, in denen säkulare Stagnation (mit $r_{n}<0$ ) eine ernste Bedrohung darstellt und angesichts der Gefahr von Deflation auch $\pi^{e}$ abfällt oder gar negativ wird, droht Geldpolitik wirkungslos zu werden.

\section{Anhebung des Inflationsziels?}

Lange Zeit herrschte die Einschätzung vor, die Zinsuntergrenze wirke nur äußerst selten als ernste Restriktion, und dann allenfalls nur für kurze Zeit. Diese Einschätzung basierte vor allem auf Simulationen anhand der historisch ruhigen Zeiten der „Großen Mäßigung“ in den 1980er und 1990er Jahren. Die Erfahrungen nach der Finanzkrise haben hier jedoch zu einem drastischen Umdenken geführt. So geht die Forschungsabteilung der Fed mittlerweile davon aus, dass die Zinsuntergrenze im Laufe zukünftiger Rezessionen fast immer bindend werden könnte - mit möglicherweise langanhaltenden Folgen einer kostspieligen Unterauslastung von Ressourcen. 
Bei der derzeitigen Überprüfung ihrer geldpolitischen Strategie steht für die Fed die Diskussion im Fokus, wie sich das Problem der Zinsuntergrenze durch unterschiedliche Optionen abmildern lässt. ${ }^{5}$ Ein naheliegender Weg läge in der Anhebung des Inflationsziels. Je höher $\pi^{e}$, desto seltener wird schließlich die Zinsuntergrenze bindend. Standardmodelle säkularer Stagnation zeigen, dass häufig multiple Gleichgewichte auftreten: Sind die Inflationserwartungen hinreichend hoch, lässt sich ein Steady State ohne Unterauslastung der Ressourcen realisieren. Sind die Inflationserwartungen dagegen zu niedrig, kann die Wirtschaft dauerhaft im Zustand der Unterauslastung verharren. ${ }^{6}$ Wie die Erfahrung der Bank of Japan zeigt, dürfte es aber kaum möglich sein, allein durch das Verkünden eines höheren Inflationsziels die Erwartungen der privaten Wirtschaftsakteure in die gewünschte Richtung zu lenken.

Die aktuelle Diskussion innerhalb der Fed konzentriert sich deshalb darauf, durch Präzisierung des aktuellen Inflationsziels der Öffentlichkeit die Bereitschaft glaubwürdiger zu machen, die gewünschte Inflationsrate zu realisieren. Denkbare Optionen dafür bietet der Wechsel zu einem Preispfad- oder nominalem BIP-Ziel, die Betonung einer symmetrischen Bandbreite um den angestrebten Zielwert oder die Verpflichtung, zukünftig zumindest temporär ein ÜberschieBen der Inflationsraten über die Zielgröße anzustreben, um sicherzustellen, dass der Zielwert selbst bei länger anhaltenden Abweichungen im Durchschnitt letztlich dann doch erreicht wird.

\section{Niedrige Inflationserwartungen}

Trotz des massiven Einsatzes zahlreicher unkonventioneller Maßnahmen der Geldpolitik verharren nicht nur die Inflationsraten, sondern auch die Inflationserwartungen über die kommenden Jahre in vielen Industriestaaten seit Jahren unter der angestrebten Zielgröße. Bei den meisten Zentralbanken liegt diese Zielgröße bei exakt $2 \%$. Im Euroraum ist die Sache etwas komplizierter. Im Oktober 1998 definierte der EZB-Rat Preisstabilität als „eine jährliche Wachstumsrate des harmonisierten Verbraucherpreisindex für den Euroraum von mittelfristig unter $2 \%$." Als sich zu Beginn des neuen Jahrtausends weltweit die Furcht vor ,japanischen“ Verhältnissen mit der Gefahr anhaltender Deflation immer stärker ausbreitete, sah sich die EZB immer mehr mit dem Vorwurf der Asymmetrie konfrontiert: Die ursprüngliche Definition lasse offen, ob die EZB auch bereit sei, einer zu niedrigen Inflationsrate entschieden entgegenzusteuern, um das Entstehen einer deflationären Spirale zu verhindern. Bei

5 Vgl. dazu Federal Open Market Committee (FOCM): Minutes of the Federal Open Market Committee, 17.-18.9.2019, https://www.federalreserve.gov/monetarypolicy/files/fomcminutes20190918.pdf (10.1.2020).

6 Vgl. etwa G. Illing et al., a.a. O. einer Revision der geldpolitischen Strategie präzisierte der EZB-Rat daraufhin im Mai 2003 die Definition der Preisstabilität als Inflation von unter, aber nahe $2 \%$.

Während die meisten anderen Zentralbanken mit einer symmetrischen Zielgröße arbeiten, ließ auch die damalige Präzisierung offen, was mit dieser Definition konkret gemeint sein könnte. Bei der Vorstellung der revidierten Strategie am 8. Mai 2003 erläuterte Otmar Issing, damals Chefvolkswirt der EZB, es gehe darum, die Inflationserwartungen unterhalb von $2 \%$ zu verankern - etwa in einer Bandbreite zwischen $1,7 \%$ bis $1,9 \%$.

Im Gegensatz dazu betonte Mario Draghi in seinem letzten Amtsjahr als EZB-Präsident mehrmals (etwa in seiner Rede "Twenty Years of the ECB's monetary policy“ auf der EZB-Forschungskonferenz in Sintra im Juni 2019), ${ }^{7}$ die angestrebte Inflationsrate von unter, aber nahe $2 \%$ sei völlig symmetrisch; diese Symmetrie bedeute nicht nur, dass die EZB keine persistent zu niedrige Inflationsrate akzeptieren werde; die mittelfristige Perspektive impliziere vielmehr auch, dass der Wert von $2 \%$ keine Obergrenze darstelle: Temporäre Abweichungen würden in beide Richtungen (also auch nach oben) toleriert, solange die Inflation auf mittlere Frist wieder auf die Zielgröße von knapp $2 \%$ zurückkehrt.

Auf den ersten Blick scheint der Unterschied zwischen den beiden Interpretationen eher marginal zu sein. Doch welche Sicht angemessen ist, ist entscheidend für die Frage, ob der aktuelle geldpolitische Kurs der EZB adäquat ist. Im Lauf des Jahres 2019 ist nicht nur die Inflationsrate im Euroraum wieder zurückgegangen, auch die im Verlauf der nächsten fünf Jahre erwartete Inflationsrate ist gesunken (vgl. Abbildung 4): Dem von der EZB durchgeführten Survey of Professional Forecasters (SPF) zufolge lagen die Inflationserwartungen über die nächsten fünf Jahre im Dezember 2019 bei 1,7\%; gemessen anhand von Marktdaten aus inflationsindexierten Swaps lagen sie dagegen im September 2019 bei ca. 0,8\%, nachdem sie von 0,5\% Anfang 2015 (dem Beginn des Programms quantitativer Lockerung der EZB) bis auf ca. 1,5\% im Sommer 2018 angestiegen waren. Sieht man im aktuellen Rückgang keinen Grund zur Besorgnis (vielleicht eher sogar den Aufbruch traumhafter Zeiten absoluter Preisstabilität), gibt es keine Rechtfertigung für die Fortsetzung der lockeren Geldpolitik. Deutet man den Rückgang der Inflationserwartungen dagegen als Indiz für die Gefahr, die effektive Zinsuntergrenze könnte im nächsten Konjunkturabschwung bald wieder bindend werden, wäre ein entschiedenes Gegensteuern angebracht.

7 M. Draghi: Twenty Years of the ECB's monetary policy, Sintra 2019, https://www.ecb.europa.eu/press/key/date/2019/html/ecb. sp190618 ec4cd2443b.en.html (9.1.2020). 
Abbildung 4

Rückgang der Inflationserwartungen im Euroraum

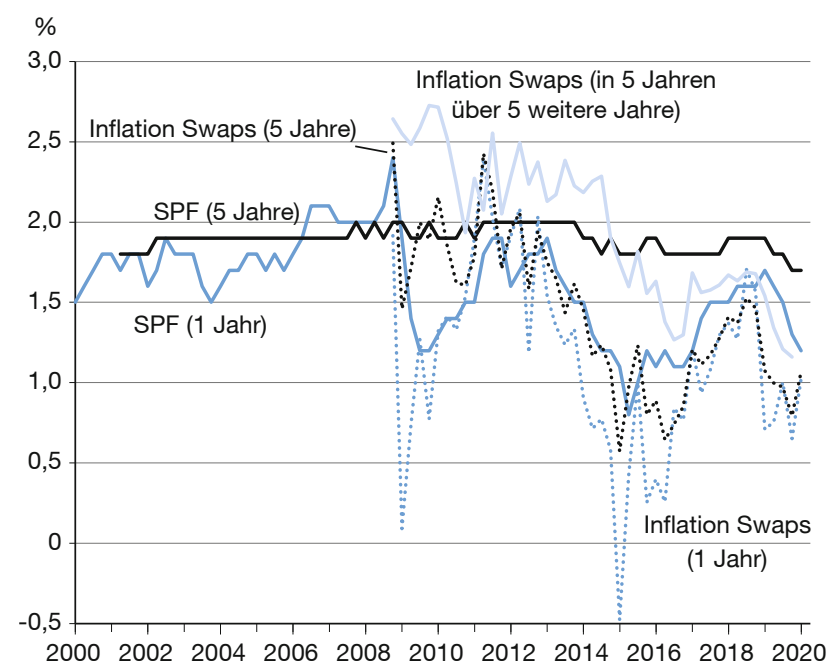

Quelle: EZB: Survey of Professional Forecasters (SPF); Thomson Reuters (Inflation Swaps).

\section{Präzisierung der angestrebten Zielinflationsrate}

Ähnlich wie in den USA dürfte die Präzisierung der angestrebten Zielinflationsrate auch bei der 2020 vorgesehenen neuerlichen Überprüfung der geldpolitischen Strategie der EZB eine zentrale Rolle spielen. Dabei ist zu hoffen, dass die von der Fed gewonnenen Erkenntnisse auch im Euroraum ernst genommen werden. Die Klarstellung, dass die EZB ein völlig symmetrisches Inflationsziel um den Zielwert von $2 \%$ anstrebt, könnte ein wichtiges Signal setzen. Dabei erscheint zweitrangig, ob dies durch den Wechsel zu einer Bandbreite oder durch die Verpflichtung auf ein temporäres Überschießen erfolgt.

Schließlich muss es darum gehen, zukünftig den Handlungsspielraum so zu erweitern, dass auch in einem Niedrigzins-Umfeld hinreichende Munition für eine aktive Stabilisierungspolitik gewährleistet ist. Dabei bleiben wenig Optionen: Der verstärkte Einsatz unkonventioneller geldpolitischer Maßnahmen; die Neubelebung aktiver Fiskalpolitik als Konjunkturinstrument oder aber eine glaubwürdige Strategie, zukünftig zumindest temporär ein Überschießen der Inflationsraten über die Zielgröße zu tolerieren.

Es erscheint geradezu paradox, dass ausgerechnet die schärfsten Kritiker der unkonventionellen Geldpolitik genau diejenigen sind, die auch alle naheliegenden Alternativen vehement ablehnen: Den verstärkten Einsatz der Konjunkturpolitik ebenso wie temporär höhere Inflationsraten. Werden diese Optionen verworfen, bleibt nur die Fortsetzung oder gar Ausweitung massiver unkonventioneller Maßnahmen mit der Gefahr unbeabsichtigter Nebenwirkungen; sollte dies nicht helfen, dürfte der Druck immer stärker werden, Zuflucht zu noch weit unkonventionelleren Maßnahmen wie etwa dem Drucken von Helikoptergeld zu suchen. 